\title{
Potentielle Erfolgsindikatoren für die Durchführung von Projekten zur Arzt-Apotheker-Zusammenarbeit - eine systematische Übersicht
}

\author{
Potential Benchmarks for Successful Interdisciplinary \\ Collaboration Projects in Germany: A Systematic Review
}

\author{
Autoren \\ Marina Weißenborn' ${ }^{1}$, Martin Schulz² , Manuel Kraft ${ }^{1}$, Walter E. Haefeli ${ }^{1}$, Hanna M. Seidling1
}

Institute

1 Abteilung Klinische Pharmakologie und Pharmakoepidemiologie, Kooperationseinheit Klinische Pharmazie, Universitätsklinikum Heidelberg, Heidelberg

2 ABDA - Bundesvereinigung Deutscher Apothekerverbände e. V., Geschäftsbereich Arzneimittel, Berlin

Schlüsselwörter

Übersichtsarbeit, Ärzte, Apotheker, Zusammenarbeit, Erfolgsindikatoren

Key words

review, physicians, pharmacists, collaboration, performance indicators

Bibliografie

DOI https://doi.org/10.1055/a-0592-7184

Online-Publikation: 21.6.2018

Gesundheitswesen 2019; 81: 1057-1068

(C) Georg Thieme Verlag KG Stuttgart · New York

ISSN 0941-3790

Korrespondenzadresse

PD Dr. Hanna M. Seidling

Abteilung Klinische Pharmakologie und

Pharmakoepidemiologie

Kooperationseinheit Klinische Pharmazie

Universitätsklinikum Heidelberg

Medizinische Klinik

69120 Heidelberg

Hanna.Seidling@med.uni-heidelberg.de

\section{ZUSAMMENFASSUNG}

Ziel der Studie Die Zusammenarbeit von Ärzten und Apothekern ist essentiell, um eine effektive und sichere Patientenbetreuung zu gewährleisten. Insbesondere im ambulanten Sektor ist die Zusammenarbeit jedoch nicht standardisiert und unterschiedlich intensiv. In dieser Arbeit sollen daher Projekte zur interdisziplinären Zusammenarbeit aus dem ambulanten Versorgungssektor in Deutschland vorgestellt und vielversprechende Ansätze aufgezeigt werden, die bei der Durchführung zukünftiger Projekte Beachtung finden sollten.
Methode Im Rahmen einer systematischen Literaturrecherche wurden unter Berücksichtigung der PRISMA-Guideline Projekte identifiziert, deren zentraler Bestandteil die ArztApotheker-Zusammenarbeit ist.

Ergebnisse Die Literatursuche ergab 542 passende Artikel. Insgesamt wurden 6 potentielle Erfolgsindikatoren zur Durchführung von Projekten zur Arzt-Apotheker-Zusammenarbeit identifiziert: Persönliches Kennenlernen (I), das Einbeziehen beider Heilberufler in die Projektplanung (II), die Etablierung gemeinsamer (Informations-) Veranstaltungen (III), die Sicherstellung der (technischen) Machbarkeit (IV) durch Incentivierung $(\mathrm{V})$ sowie die Einbindung in bestehende Versorgungsstrukturen (VI).

Schlussfolgerung Insgesamt wurden nur wenige Studien wissenschaftlich publiziert. Die Zusammenarbeit selbst wird meist nicht standardisiert, lückenhaft oder nur einseitig erfasst und kann daher nicht umfassend bewertet werden. Erfolgreiche Arzt-Apotheker-Kooperationen zeichneten sich häufig durch langjährige, persönliche Kontakte aus. Interdisziplinäre Lehrveranstaltungen während der universitären Ausbildung könnten daher bereits früh Sympathie und gegenseitiges Verständnis fördern. Um eine kontinuierliche Evaluation der Zusammenarbeit und eine Vergleichbarkeit verschiedener Projekte zu gewährleisten, ist der Einsatz standardisierter Messwerkzeuge in zukünftigen Studien unbedingt erforderlich.

\section{ABSTRACT}

Aim of the study Collaboration between general practitioners and community pharmacists is essential to ensure safe and effective patient care. However, collaboration in primary care is not standardized and varies greatly. This review aims to highlight projects about professional collaboration in ambulatory care in Germany and identifies promising approaches and successful benchmarks that should be considered for future projects.

Methods A systematic literature search was performed based on the PRISMA guidelines to identify articles focusing on professional collaboration between general practitioners and pharmacists.

Results A total of 542 articles were retrieved. Six potential premises for successful cooperation projects were identified: 
GP and CP knowing each other (I), involvement of both health care providers in the project planning (II), sharing of experience or concerns during regular joint meetings enabling continuing evaluation and adaption (III), ensuring (technical) feasibility (IV), particularly by providing incentives (V), and by integrating these projects into existing health care structures ( $\mathrm{VI}$ ).

Conclusion Only few studies have been published in scientific journals. There was no standardized assessment of how the participants perceived their collaboration and how it facilitates their daily work, even when the study aimed to evaluate GP-CP collaboration. Successful cooperation between GP and CP in daily routine care was often characterized by personal contact and longtime relationships. Therefore, collaborative teaching sessions at university might establish sympathy and mutual understanding right from the beginning. There is a strong need to establish standardized tools to evaluate collaboration in future projects and to enable comparability of different studies.

\section{Einleitung}

Eine enge und wohlkoordinierte Zusammenarbeit von Ärzten und Apothekern ist essentiell, um eine optimale und effektive Betreuung von Patienten zu gewährleisten [1]. Ein kollegiales Zusammenspiel mit einer offenen, harmonischen Kommunikation erleichtert das Erfüllen organisatorischer Anforderungen, zudem trägt es zu einer Erhöhung der Arzneimitteltherapiesicherheit (AMTS) und somit zur Verbesserung von klinischen Endpunkten bei [2,3]. Hierbei bringen beide Heilberufler eine unterschiedliche, sich ergänzende Expertise ein, die - gebündelt und koordiniert - den fehleranfälligen Medikationsprozess besonders effektiv begleiten könnte.

Die Zusammenarbeit und Kommunikation zwischen den Heilberuflern in Deutschland ist jedoch, v. a. im ambulanten Versorgungssektor, unterschiedlich intensiv. Dies hängt einerseits von der Einstellung und Bereitschaft der einzelnen Personen ab, wobei unter anderem Sympathie, Vertrauen, Motivation, die Fachkompetenz der jeweiligen Heilberufler und das gegenseitige Kennenlernen eine Rolle spielen [4, 5]. Andererseits wird die Zusammenarbeit durch föderalistische und zentrale Rahmenbedingungen (z. B. steigende bürokratische Anforderungen, Zeitmangel), regionale Gegebenheiten (z. B. räumliche Distanz) und lokale Gewohnheiten (z. B. Kommunikation über Medizinische Fachangestellte/Pharmazeutisch-technische Assistenten) bestimmt [4, 5].

In einigen anderen Ländern blicken Arzt und Apotheker oftmals auf eine bereits seit vielen Jahren bestehende, enge Zusammenarbeit zurück. Dabei werden einige Projekte auf nationaler Ebene oder durch Krankenkassen gefördert, andere wiederum konnten sogar in der Routineversorgung etabliert werden. In den Niederlanden bspw. nehmen regelhaft alle niedergelassenen Ärzte und Apotheker an regelmäßig stattfindenden, interdisziplinären Qualitätszirkeln teil [6]. In Australien und England führen Ärzte und Apotheker gemeinsam die sogenannten „Home Medicines Reviews“ bzw. „Medicines Use Reviews “ für ihre Patienten durch, während in Deutschland gerade erste Bemühungen hin zu einem interdisziplinären Medikationsmanagement unternommen werden [7, 8]. In England und Kanada können Apotheker als „prescribing pharmacists“ sogar in den Verschreibungsprozess einbezogen werden $[9,10]$.

Solche Projekte zeigen, dass eine Zusammenarbeit in der Routine erfolgreich verlaufen kann. Aufgrund der sehr unterschiedlichen Strukturen, Gesundheits- und Ausbildungssysteme können solche Erfahrungen zum jetzigen Zeitpunkt jedoch nur bedingt auf deutsche Verhältnisse übertragen werden.
Daher soll diese Arbeit eine umfassende Übersicht zum aktuellen Stand der interdisziplinären Zusammenarbeit zwischen Ärzten und Apothekern im ambulanten Versorgungssektor in Deutschland geben, wobei besonders erfolgsversprechende bzw. gescheiterte Ansätze hervorgehoben werden. Ein weiterer Schwerpunkt liegt in der Identifizierung von Erfolgsindikatoren bzw. Gründen für Misserfolge, die für die Planung und Durchführung zukünftiger Projekte und Studien berücksichtigt werden sollten. Dabei werden auch einige kleinere, lokale Projekte einbezogen, die zwar nicht wissenschaftlich publiziert wurden, die jedoch oftmals ein erster Schritt zu einem engeren Miteinander sind.

\section{Methoden}

Von Juli 2016 bis Januar 2017 wurde unter Berücksichtigung der PRISMA-Guideline eine umfassende Literaturrecherche durchgeführt [11].

Für die systematische Suche in den Literaturdatenbanken PubMed und Psyclnfo wurde anhand eines adaptierten PICO Schemas eine umfassende Suchstrategie erarbeitet ( $>$ Tab. 1). Die Literatursuche in deutschsprachigen Fachzeitschriften, Internetportalen, dem Deutschen Register Klinischer Studien (DRKS) und dem Onlineportal German Medical Science (GMS) wurde in mehreren Einzelschritten durchgeführt, da die strukturierte Verknüpfung verschiedener Suchbegriffe hier nicht unterstützt wird ( $\triangleright$ Tab. 1).

Alle 17 deutschen (Landes-)Apothekerkammern sowie ausgewählte ärztliche Fachgesellschaften (Deutsche Gesellschaft für Allgemein- und Familienmedizin DEGAM, Atemwegsliga, Deutsche Diabetes Gesellschaft DDG, Deutsche Schmerzgesellschaft, Deutsche Gesellschaft für Neurologie, für Pneumologie und Beatmungsmedizin und für Psychosomatische Medizin und Ärztliche Psychotherapie) wurden angeschrieben. Zudem wurden die Projektdatenbanken einschlägiger Stiftungen zur Pharmazeutischen Betreuung bzw. klinischen Pharmazie (Apothekerstiftung Westfalen-Lippe, BA KlinPharm, Dr. August und Dr. Anni Lesmüller Stiftung, WIPIG, Förderinitiative Pharmazeutische Betreuung e.V.) und die des Innovationsfonds gescreent. Für geplante Initiativen auf Bundesebene wurde die Homepage des Aktionsplans zur Verbesserung der Arzneimitteltherapiesicherheit in Deutschland, initiiert durch das Bundesministerium für Gesundheit, durchsucht. Zuletzt wurden die Programmhefte und Abstracts relevanter deutscher Kongresse (DEGAM-Kongress, Deutscher Kongress für Versorgungsforschung DKVF, Gesellschaft für Arzneimittelanwendungsforschung und Arz- 
- Tab. 1 Suchstrategie.

\begin{tabular}{|c|c|c|c|c|c|c|}
\hline \multicolumn{7}{|l|}{ Pubmed-Suchstrategie: } \\
\hline $\begin{array}{l}\text { ‘germany’[MeSH] OR ‘germany’ OR } \\
\text { ‘german’ }\end{array}$ & AND & $\begin{array}{l}\text { 'interdisciplinary' OR 'multidisciplinary' } \\
\text { OR 'interprofessional' OR 'interpersonal' } \\
\text { OR 'communication' OR 'cooperation' } \\
\text { OR 'teamwork' OR 'relation' OR 'Health } \\
\text { Communication'[MeSH] OR } \\
\text { 'Communication'[MeSH] OR 'Interdisci- } \\
\text { plinary Communication'[MeSH] OR } \\
\text { 'Interpersonal Relations'[MeSH] OR } \\
\text { 'Cooperative Behavior'[MeSH] OR } \\
\text { 'Interprofessional Relations'[MeSH] }\end{array}$ & AND & pharmacist & AND & $\begin{array}{l}\text { 'general } \\
\text { practitioners'[MeSH] OR } \\
\text { 'general practitioners' }\end{array}$ \\
\hline \multicolumn{7}{|l|}{ Psyclnfo: } \\
\hline germany OR german & AND & $\begin{array}{l}\text { interdisciplinary OR multidisciplinary OR } \\
\text { interprofessional OR interpersonal OR } \\
\text { communication OR cooperation OR } \\
\text { teamwork OR relation OR Health } \\
\text { Communication OR Interdisciplinary } \\
\text { Communication OR Interpersonal } \\
\text { Relations OR Cooperative Behavior }\end{array}$ & AND & pharmacist & AND & $\begin{array}{l}\text { general practitioner OR } \\
\text { practitioner OR physician }\end{array}$ \\
\hline \multicolumn{7}{|c|}{ Fachzeitschriften, Internetportale, DRKS (deutschsprachig): } \\
\hline- & & $\begin{array}{l}\text { Kooperation ODER Kommunikation } \\
\text { ODER Zusammenarbeit }\end{array}$ & UND & Apotheker & UND & Arzt ODER Ärzte \\
\hline \multicolumn{7}{|c|}{ Der Hausarzt, Portal German Medical Science (deutschsprachig): } \\
\hline- & & - & & Apotheke $^{1}$ & & - \\
\hline \multicolumn{7}{|c|}{ Kongresse - Programmhefte und Abstracts (nicht verknüpfte Stichwortsuche, deutsch- und englischsprachig): } \\
\hline- & & Kommunikation ODER Zusammenarbeit & & \multicolumn{3}{|c|}{ Apotheke ODER pharmac } \\
\hline
\end{tabular}

neimittelepidemiologie GAA, Jahrestagung Aktionsbündnis für Patientensicherheit APS, Kongress für Patientensicherheit bei medikamentöser Therapie) aus den letzten 2 Jahren (2016 und 2017) durchsucht.

Eingeschlossen wurden Artikel und Projekte, die sich mit der Arzt-Apotheker-Zusammenarbeit im ambulanten Versorgungssektor in Deutschland befassen (im 4-Augen-Prinzip).

\section{Ergebnisse}

In den Volltextdatenbanken und Onlinequellen wurde eine fünfstellige Anzahl an Treffern gescreent und 578 thematisch passende Artikel ausgelesen ( $\triangleright$ Tab. 2). Insgesamt 14 Kammern (16 Rückmeldungen) und 2 Fachgesellschaften (5 Rückmeldungen) berichteten von gemeinsamen Fortbildungsveranstaltungen oder Projekten zur Förderung der Zusammenarbeit - ebenso die Projektdatenbanken verschiedener Stiftungen und des Innovationsfonds. Die Treffer wurden in die folgenden 4 Kategorien eingeteilt:

\section{Stellungnahmen und Kurzberichte}

In Tagungsberichten wurden in den vergangenen drei Jahren v. a. die Themen Medikationsplan, Medikationsanalysen, Medikationsmanagement und e-Health diskutiert (z. B. $[12,13])$. Dabei nahmen Ärzte und Apotheker Stellung, welcher Heilberufler welche Aufgaben übernehmen sollte und welche Aufgaben welche Kompetenzen erfordern. Nur selten wurden konkrete Schritte zur Aufteilung der Verantwortlichkeiten vorgeschlagen, was eine Verwendung dieser Artikel für die Erarbeitung von Konzepten zur Arzt-Apotheker-Zusammenarbeit erschwert.

\section{Theoretische Modelle}

Während die Arzt-Apotheker-Zusammenarbeit und deren Einflussfaktoren international häufig anhand theoretischer Stufenmodelle beschrieben wird (z. B. mittels des Collaborative Working Relationship-Modells), gibt es solche Kooperationsmodelle bisher nicht für Deutschland [14]. Stattdessen wurden Modelle zur Darstellung von Zuständigkeiten und Verantwortlichkeiten entwickelt. So sollten sich Ärzte, Apotheker und Pflegekräfte der Universität Bonn gegenseitig Aufgabenfelder in der Patientenversorgung zuordnen (z. B. Arzneimittelapplikation, Dosierungsberechnungen, Patientenaufklärung) [15]. Daraus entstanden ist das „multiprofessional cancer medication management“-Modell [15]. Im ARMIN-Projekt wurde eine konkrete Arbeitsaufteilung ( $\triangleright$ Tab. 3) und Prozessgestaltung für eine kooperative Patientenversorgung ausgearbeitet und vertraglich konsentiert [16].

\section{Projekte und Initiativen}

- Tab. 4 gibt eine Übersicht über Projekte und Initiativen, die (noch) nicht systematisch wissenschaftlich begleitet werden und die vielmehr aus der Praxis der gemeinsamen Arbeit entstanden sind. Sie lassen sich grob in 2 Kategorien einordnen:

Zum einen wird eine Intensivierung der Zusammenarbeit bspw. durch gemeinsame Aus- oder Fortbildungsveranstaltungen angestrebt. Zum anderen werden konkrete Interventionen geplant (z. B. Durchführung einer Medikationsanalyse), bei denen untersucht wird, inwieweit deren Outcome durch die Zusammenarbeit von Arzt und Apothekern verbessert werden kann. Die Initiatoren dieser Initiativen sind vielfältig und reichen von Universitäten (Hospitationen, Arbeitsgemeinschaft InterAG $[17,18]$ ) über Kammern 
und Vereine (z. B. Aktionsbündnis Patientensicherheit, interdisziplinäre Fortbildungen, Positionspapiere [19-22]) bis hin zu lokalen Zusammenschlüssen von Ärzten und Apothekern, die sich über die Betreuung bzw. Behandlung ihrer Patienten austauschen (z. B. Betreuung von Diabetes-Patienten [23]). Aus einigen dieser Initiativen kann man inzwischen eine jahrelange gemeinsame Historie ablesen, aus der z. T. konkrete Folgeprojekte hervorgingen (z. B. Präventionsaktion „Fit-für-fünf-km“ [24], Standardisierung von Rezepturen [25]).

Des Weiteren wurden 45 Projekte identifiziert, die wissenschaftlich evaluiert wurden. Diese Projekte werden in > Tab. $\mathbf{5}$ inhaltlich vorgestellt und in Bezug auf die interdisziplinäre Zusammenarbeit bewertet.
Die durch den Innovationsfond geförderten Projekte, bei denen die Arzt-Apotheker-Zusammenarbeit einen zentralen Aspekt darstellt, laufen gerade an und können in dieser Arbeit noch nicht berichtet werden (z. B. „HIOPP 3“) [26]. Auch im Aktionsplan Arzneimitteltherapiesicherheit sind (allgemeine) Maßnahmen zur Förderung der Arzt-Apotheker-Zusammenarbeit formuliert, jedoch sind die entsprechenden Maßnahmen erst für die kommenden Jahre geplant bzw. die Ergebnisse noch nicht publiziert [27].

\section{Materialien und Messinstrumente}

Unter anderem im Rahmen des Qualitätsmanagements wurden standardisierte Dokumentationsvorlagen entwickelt, um den In-

\Tab. 2 Übersicht über die Gesamtanzahl der Treffer und der jeweils thematisch passenden Artikel.

\begin{tabular}{|c|c|c|c|}
\hline Informationsquelle & $\begin{array}{l}\text { Anzahl Treffer insgesamt } \\
\text { (bis 01/2017) }\end{array}$ & $\begin{array}{l}\text { Davon ausgelesener } \\
\text { Zeitraum }\end{array}$ & $\begin{array}{l}\text { Darin thematisch } \\
\text { passend }\end{array}$ \\
\hline \multicolumn{4}{|c|}{ Literaturdatenbanken } \\
\hline Pubmed & 88 & bis $01 / 2017$ & 11 \\
\hline Psyclnfo & 17 & bis $01 / 2017$ & 1 \\
\hline GMS & 202 & bis $10 / 2017$ & 24 \\
\hline \multicolumn{4}{|c|}{ Deutschsprachige Fachzeitschriften } \\
\hline Deutsche Apotheker Zeitung & 1824 & bis $01 / 2017$ & 125 \\
\hline Pharmazeutische Zeitung + PTA-Forum & 1491 & bis $12 / 2016$ & 138 \\
\hline Deutsches Ärzteblatt & 2680 & $01 / 2010$ bis $01 / 2017$ & 82 \\
\hline Der Hausarzt & 86 & bis $01 / 2017$ & 5 \\
\hline \multicolumn{4}{|c|}{ Deutschsprachige Internetportale } \\
\hline Apotheke Adhoc & 3574 & bis $01 / 2017$ & 149 \\
\hline Ärztezeitung & 541 & $01 / 2010$ bis $01 / 2017$ & 25 \\
\hline \multicolumn{4}{|c|}{ Sonstige } \\
\hline DRKS & 279 & bis $01 / 2017$ & 6 \\
\hline \multicolumn{4}{|c|}{ Kongresse und Jahrestagungen } \\
\hline DEGAM-Kongress & \multirow{5}{*}{$\begin{array}{l}\text { Durchsuchen von Abstract- und } \\
\text { Programmheften }{ }^{1}\end{array}$} & \multirow{5}{*}{$2016-2017^{2}$} & 6 \\
\hline DKVF & & & 4 \\
\hline GAA-Jahrestagung & & & 2 \\
\hline APS-Jahrestagung & & & 0 \\
\hline \multirow[t]{2}{*}{ Patientensicherheit bei medikamentöser Therapie } & & & 0 \\
\hline & & & $\sum=578$ \\
\hline
\end{tabular}

\Tab. 3 Aufteilung von Zuständigkeiten am Beispiel des ARMIN-Projektes gemäß der Leistungsbeschreibungen im Medikationsmanagement (Geschäftsbereich Arzneimittel der ABDA).

\begin{tabular}{|c|c|}
\hline Apotheker & Arzt \\
\hline $\begin{array}{l}\text { - Ersterfassung Arzneimittel/Brown Bag }{ }^{1,2} \\
\text { - Pharmazeutische AMTS-Prüfung1 } \\
\text { - Medikationsplan - over-the-counter Arzneimittel (Selbstmedikation) und } \\
\text { Handelsnamen der auf Rezept abgegebenen AM } \\
\text { - Beratungsthemen: } \\
\text { - arzneimittelbezogenes Wissen und praktische Umsetzung der Therapie } \\
\text { - Unbewusste Non-Adhärenz } \\
\text { - Neue Arzneimittel/Umstellungen } \\
\text { - Detektion von Versorgungslücken mit gezielten Interventionen }\end{array}$ & $\begin{array}{l}\text { - Multimedikationspriorisierung } \\
\text { - Medizinische AMTS-Prüfung } \\
\text { - } \\
\text { - Medikationsplan - verschreibungspflichtige und verordnungsfähige } \\
\text { over-the-counter Arzneimittel } \\
\text { - Beratungsthemen: } \\
\text { - Wissensvermittlung } \\
\text { - Bewusste Non-Adhärenz } \\
\text { - Psychische Probleme } \\
\text { - Gemeinsame Therapieplanung und -kontrolle mit Patienten (Ziele) }\end{array}$ \\
\hline
\end{tabular}


\ Tab. 4 Übersicht über interdisziplinäre Projekte und Initiativen, typischerweise ohne wissenschaftliche Begleitevaluation.

\begin{tabular}{|c|c|c|c|}
\hline Übersicht & Beispielhafte Projekt (Beteiligte/Veranstaltungsort) & Zeitraum & Referenz \\
\hline \multirow{9}{*}{$\begin{array}{l}\text { Arbeitskreise, Fortbildungen, } \\
\text { Qualitätszirkel, Informations- } \\
\text { veranstaltungen }\end{array}$} & $\begin{array}{l}\text { - Verschiedene Apotheker-/Ärztekammern, ärztliche Fachgesellschaften, } \\
\text { Berufsverbände, z. B.: }\end{array}$ & - & Kontakt \\
\hline & - Verbesserung der Qualität dermatologischer Rezepturverordnungen (BAK, BVDD) & Seit 2008 & [46] \\
\hline & - Brandenburgische Apotheker- und Ärztetage (LAK, LÄK BB) & Seit 2014 & [47] \\
\hline & $\begin{array}{l}\text { - Interdisziplinäre Experteninterviews: Interventionskonzept zur Förderung des } \\
\text { Selbstmanagements bei chronisch Kranken (Universität Bielefeld) }\end{array}$ & $2004-2006$ & [48] \\
\hline & $\begin{array}{l}\text { - Gesundheitstage: Arzt-Apotheker-Dialog, Förderung der interdisziplinären Kommuni- } \\
\text { kation, Schnittstellenoptimierung }\end{array}$ & 2016 & [49] \\
\hline & - Interdisziplinäre Informationsveranstaltungen zum Medikationsplan (AKNR, ÄKNO) & Seit 2016 & [50] \\
\hline & $\begin{array}{l}\text { - Sapremo: Interprofessionelle Workshops und Fortbildungen (Universitätsklinikum } \\
\text { Halle, Ärztekammer SA, Apothekerkammer SA u. a.) }\end{array}$ & $2016-2017$ & [51] \\
\hline & $\begin{array}{l}\text { - Interdisziplinäre Fokusgruppen: Absetzstrategien bei Patienten mit Polypharmazie } \\
\text { (Universität Marburg, Medizinische Hochschule Hannover) }\end{array}$ & 2017 & [52] \\
\hline & $\begin{array}{l}\text { - Interdisziplinäre Fokusgruppen: AMTS-Verbesserung bei Pflegeheimbewohnern mit } \\
\text { chronischer Niereninsuffizienz (IPP Bremen, Universität Oldenburg) }\end{array}$ & 2017 & [53] \\
\hline \multirow{6}{*}{$\begin{array}{l}\text { Projekte von bzw. für } \\
\text { Pharmazie- und Medizin- } \\
\text { studierende }\end{array}$} & $\begin{array}{l}\text { - Teddybärenkrankenhaus für Kinder (verschiedene Standorte, Team aus Humanmedizin-l } \\
\text { Zahnmedizin- und Pharmaziestudierenden) }\end{array}$ & Seit 2001 & [54] \\
\hline & $\begin{array}{l}\text { - Pharmaweekend: Fortbildungskongress, seit } 2009 \text { werden auch Medizinstudierende } \\
\text { eingeladen (BPhD e.V., Fachschaften) }\end{array}$ & Seit 2003 & [55] \\
\hline & $\begin{array}{l}\text { - 1. Bundeskongress der Medizinstudierenden: Einladung von Pharmaziestudierenden zu } \\
\text { interdisziplinären Workshops (bvmd) }\end{array}$ & 2009 & [56] \\
\hline & $\begin{array}{l}\text { - Gründung Arbeitsgruppe „InterAG“: Meinungspapier zur Intensivierung der Zusam- } \\
\text { menarbeit von Studierenden der Gesundheitsberufe (BPhD e.V.) }\end{array}$ & Seit 2014 & [18] \\
\hline & $\begin{array}{l}\text { - Hospitation von Medizinstudenten in der Apotheke: Kennenlernen der Hürden in der } \\
\text { AM-Abgabe (Magdeburg, OVGU, AK SA) }\end{array}$ & Seit 2016 & [17] \\
\hline & - Modellstudiengang Pharmazie (Universität Leipzig) & In Planung & [32] \\
\hline \multirow{8}{*}{$\begin{array}{l}\text { Interdisziplinäre Projekte aus } \\
\text { Apotheken und Arztpraxen }\end{array}$} & $\begin{array}{l}\text { - Hospitation eines Arztes in der Apotheke zum Kennenlernen des Arbeitsalltags in der } \\
\text { Offizin (Augsburg) }\end{array}$ & 2000 & [57] \\
\hline & $\begin{array}{l}\text { - Betreuung von Diabetespatienten, Fortbildungsakademie - ausgezeichnet mit dem } \\
\text { Springer-Medizin-Preis „bestes Arzt-Apotheker-Team“ (Warburg) }\end{array}$ & Seit 2003 & [23] \\
\hline & $\begin{array}{l}\text { - Präventionsaktion „Fit für fünf km“ - gemeinsame Lauftreffen für Patienten, } \\
\text { Organisation der „Erftstädter Gesundheitstage“ (Erftstadt) }\end{array}$ & Seit vor 2009 & [24] \\
\hline & $\begin{array}{l}\text { - Interdisziplinäre Ernährungsberatung, Lebensstilumstellung und physiotherapeutisches } \\
\text { Bewegungsprogramm (Schleiz) }\end{array}$ & 2009 & [58] \\
\hline & $\begin{array}{l}\text { - Absprache von Therapieempfehlungen, Impfstoffen, neuen Medikamenten, Ausarbei- } \\
\text { tung eines Diät-Konzepts (Wolfschlugen) }\end{array}$ & Seit vor 2011 & [59] \\
\hline & - Absprache zu dermatologischen Rezepturen (z. B. Oberpfalz) & $\mathrm{N} / \mathrm{A}$ & [25] \\
\hline & $\begin{array}{l}\text { - HIV-Schwerpunkt-Apotheken, z. B. Entwicklung von Checklisten zur Bestimmung der } \\
\text { Adhärenz und Feststellung einer Progredienz (z. B. Berlin) }\end{array}$ & $\mathrm{N} / \mathrm{A}$ & [60] \\
\hline & $\begin{array}{l}\text { - } 7 \text { + Projekt - AMTS-Optimierung bei Heimbewohnern, Entwicklung von Faxvorlagen } \\
\text { zur Verbesserung der Kommunikation (Gelsenkirchen) }\end{array}$ & Seit 2016 & [61] \\
\hline \multirow{8}{*}{$\begin{array}{l}\text { Projekte öffentlicher } \\
\text { Organisationen }\end{array}$} & $\begin{array}{l}\text { - IDH - Interessengemeinschaft der Heilberufe: Gemeinsame Öffentlichkeitsarbeit, } \\
\text { Meinungsaustausch (AKSH, ÄKSH, AVSH, ...) }\end{array}$ & Seit 1976 & [62] \\
\hline & $\begin{array}{l}\text { - AG Arzt/Apotheker - Entwicklung eines Leitfadens zur Zusammenarbeit von Ärzten } \\
\text { und Apothekern (ABDA und BDA) }\end{array}$ & 1998 & [63] \\
\hline & $\begin{array}{l}\text { - EADV - Kommission zur Einbindung der Apotheker in die Diabetikerversorgung: z. B. } \\
\text { Entwicklung von Arbeitsmaterialien/Arbeitsabläufen (DDG, BAK) }\end{array}$ & Seit 1998 & [64] \\
\hline & $\begin{array}{l}\text { - APS - Aktionsbündnis Patientensicherheit: Entwicklung von Handlungsempfehlungen } \\
\text { und Patienteninformationen }\end{array}$ & Seit 2005 & [19-20] \\
\hline & - Ratgeberheft Kinderstube (SLAK, SLÄK, LZKS, Sächs. Landestierärztekammer) & Seit 2009 & [65] \\
\hline & $\begin{array}{l}\text { - Bündnis für System-Compliance zur Verbesserung der AMTS (AK NDS, KVN, MHH, } \\
\text { AOK, DAK, Barmer, MSD Sharp\&Dohme, Pfizer) }\end{array}$ & 2011 & [66] \\
\hline & $\begin{array}{l}\text { - Praxistauglichkeit des bundeseinheitlichen Medikationsplans (Universitätsmedizin } \\
\text { Mainz, LAK RLP, Gesundheitsministerium RLP, TK) }\end{array}$ & 2015 & [67] \\
\hline & - Baumberger Impuls - Erstellung eines gemeinsamen Positionspapiers (AKWL, ÄKWL) & 2015 & [21] \\
\hline
\end{tabular}


- Tab. 4 Fortsetzung.

\begin{tabular}{|c|c|c|c|}
\hline Übersicht & Beispielhafte Projekt (Beteiligte/Veranstaltungsort) & Zeitraum & Referenz \\
\hline & $\begin{array}{l}\text { - Verbesserung der Erreichbarkeit notdiensthabender Ärzte/Apotheker in BW, u. a. } \\
\text { Einrichten interner Telefonnummern (KV, LAK BW) }\end{array}$ & Seit 2015 & [68] \\
\hline & - Strategiepapier für eine intensivere interdisziplinäre Zusammenarbeit (SLAK, SLÄK) & 2017 & [22] \\
\hline \multicolumn{4}{|c|}{$\begin{array}{l}\text { (Landes-) Apothekerkammer (L-)AK, (Landes-) Ärztekammer (L-)ÄK, AK Sachsen-Anhalt AK SA, ÄK Nordrhein ÄKNO, ÄK Schleswig-Holstein ÄKSH, Baden- } \\
\text { Württemberg BW, Berufsverband der Deutschen Dermatologen BVDD, Brandenburg BB, Bundesapothekerkammer BAK, Bundesverband der Pharmaziestu- } \\
\text { dierenden in Deutschland e.V. BPhD, Bundesvertretung der Medizinstudierenden bvmd, Deutsche Diabetes Gesellschaft DDG, Institut für Public Health und } \\
\text { Pflegeforschung IPP, Landeszahnärztekammer Sachsen LZKS, Otto-von-Guericke-Universität Magdeburg OVGU, Rheinland-Pfalz RLP }\end{array}$} \\
\hline
\end{tabular}

formationsaustausch zwischen Arztpraxis und Apotheke zu erleichtern [28, 29]. International eingesetzte Messinstrumente zur Evaluation von Häufigkeit und Nützlichkeit der Zusammenarbeit bzw. der gegenseitigen Einstellung wurden bislang zwar übersetzt, sie werden jedoch noch nicht umfangreich eingesetzt [30].

\section{Diskussion}

Anders als erwartet wird die Zusammenarbeit der Heilberufler in den beschriebenen Projekten meist nicht standardisiert sondern vielmehr ungenau, lückenhaft oder einseitig erfasst bzw. berichtet. Nur selten wird detailliert beschrieben, auf welche Art und Weise oder wie häufig die Heilberufler in den Projekten zusammenarbeiten und ob bzw. inwiefern die im Studienplan beschriebene und die in der Studiendurchführung tatsächliche stattfindende Zusammenarbeit voneinander abweichen. Auch fehlt häufig ein Feedback der Heilberufler, bspw. hinsichtlich ihrer Zufriedenheit oder Bereitschaft, das Projekt langfristig fortzuführen. Die vorliegenden Arbeiten können daher nur begrenzt hinsichtlich Durchführung und Erfolg der interdisziplinären Zusammenarbeit bewertet werden. Insbesondere für neue Konzepte wäre es jedoch wichtig zu wissen, welche Faktoren auf Prozess- oder Organisationsebene dazu beitragen, dass die Zusammenarbeit erfolgreich verläuft, so dass eine Evaluation der Arzt-Apotheker-Zusammenarbeit konsequent in den laufenden Projekten umgesetzt werden sollte.

Auf Basis der hier berichteten Projekte lassen sich die folgenden Anhaltspunkte für eine erfolgreiche Zusammenarbeit herausarbeiten - dabei werden auch bisher nicht abgeschlossene Projekte (wie bspw. ARMIN) berücksichtigt, wenn entsprechende Zwischenergebnisse berichtet wurden.

Bei den lokalen Initiativen verliefen insbesondere diejenigen erfolgreich, in denen einer der beiden Heilberufler persönlich auf den anderen zugegangen ist [23-25]. Solche direkten Kontakte sind schwer auf landesweite Initiativen skalierbar, lassen jedoch vermuten, dass eine berufliche Zusammenarbeit gefördert werden kann, wenn man den anderen in seiner Funktion als Heilberufler kennt und sich auf diese Weise eine gewisse Akzeptanz, gegenseitiges Vertrauen und beidseitiges Verständnis entwickelt hat [31].

Bereits während der Ausbildung sollte daher das gegenseitige Kennenlernen gefördert werden, bspw. durch gemeinsame Lehrveranstaltungen. Der geplante Modellstudiengang Pharmazie an der Medizinischen Fakultät in Leipzig wird hierzu in einigen Jahren weitere Ergebnisse liefern [32]. Dieser Kontakt sollte auch nach der Ausbildung kontinuierlich gepflegt werden. Im Berufsalltag können bspw. interdisziplinäre Veranstaltungen einen persönlichen und fachlichen Austausch ermöglichen (z. B. zu gesetzlichen Rah- menbedingungen oder Herausforderungen im Praxisalltag). Eine solche Verstetigung des interdisziplinären Austausches auch unabhängig von konkreten Patientenfällen wurde z. B. durch die standardisierte Einführung von interdisziplinären Qualitätszirkeln in den Niederlanden erreicht [6].

Auf regionaler Ebene erscheinen v. a. jene Projekte erfolgreich, in denen Ärzte und Apotheker gleichermaßen in die Planung und Durchführung einbezogen werden, wie z. B. bei InTherAKT [33]. Durch die Möglichkeit zur Mitgestaltung steigt die Motivation zur Teilnahme und die anschließende Akzeptanz gegenüber der Intervention. Gleichzeitig kann die Intervention frühzeitig an Alltagsbedingungen und berufsspezifische Anforderungen angepasst und die Rollen bzw. Aufgabenbereiche im Einverständnis verteilt werden, was eine anschließende Weiterführung in der Routineversorgung vereinfacht. Daher sollten auch während der Projektlaufzeit regelmäßig (Informations-) Veranstaltungen zum Austausch und Feedback-Geben stattfinden.

Eine präzise Zuteilung von Verantwortlichkeiten und eine umfassende Prozessbeschreibung ist auch im ARMIN Projekt realisiert [16]. Diese definierte Aufteilung von Aufgaben und das strukturierte Zuarbeiten findet sich auch in international erfolgreichen Initiativen wieder $[7,8]$.

Grundvoraussetzung für eine langfristige Implementierung von neuen Konzepten zur Arzt-Apotheker-Zusammenarbeit ist deren Machbarkeit, das heißt deren einfache Umsetzbarkeit und Integration in den Apotheken- und Praxisalltag. Viele der Initiativen mit hohem zusätzlichem Arbeits- bzw. Zeitaufwand und Maßnahmen, die deutlich von der Routineversorgung abwichen, wurden nach Projektende nicht wie geplant durch- bzw. fortgeführt [35, 36]. Daher sollten, insbesondere zu Beginn der Zusammenarbeit, möglichst konkrete, gemeinsam leicht zu bewältigende Aufgaben implementiert werden.

Ein wichtiges und häufig genanntes Problem in der Zusammenarbeit der Heilberufler ist das Fehlen gemeinsamer Kommunikationsplattformen bzw. die Inkompatibilität der verschiedenen Primärsoftwaresysteme [37]. Dies erschwert zum einen den direkten Informationsaustausch, aber auch das gemeinsame Führen und Pflegen einer Patientenakte, z. B. um einen gemeinsamen, wirklich vollständigen Medikationsplan zu erstellen. In einigen Projekten wurden hierfür bereits Lösungen gefunden; über die vollautomatische Datenübertragung wird bspw. in den Projekten ARMIN, InTherAKT und Arzneimittelkonto NRW positiv berichtet [16, 33, 38].

Eng mit der (technischen) Machbarkeit der Zusammenarbeit ist die finanzielle Umsetzbarkeit verbunden, die Intervention sollte insofern wirtschaftlich sein, dass ein Mehraufwand entweder an anderer Stelle zu Zeitersparnis oder Vereinfachungen führt oder pri- 
> Tab. 5 Übersicht über Projekte mit wissenschaftlicher Begleitevaluation.

\begin{tabular}{|c|c|c|c|c|c|}
\hline Titel des Projektes & Wer & Inhalt & Bewertung & Zeitraum & Referenz \\
\hline TOM-ASTHMA (1) & ABDA (ZAPP) & PB von Asthmapatienten & 1 & $1996-1998$ & [69-70] \\
\hline $\begin{array}{l}\text { PB - ältere, multimorbide } \\
\text { Patienten }\end{array}$ & $\begin{array}{l}\text { Klinische Pharmakologie, } \\
\text { Berlin }\end{array}$ & $\begin{array}{l}\text { Teil einer EU geförderten Studie in } 7 \\
\text { EU-Ländern }\end{array}$ & 2 & 1997-1999 & [71] \\
\hline PB - Schmerzpatienten & $\begin{array}{l}\text { Universität Köln, TU } \\
\text { Dresden, SLAK }\end{array}$ & $\begin{array}{l}\text { Betreuungsdesign für Schmerzpatienten im } \\
\text { Alltag }\end{array}$ & 2 & $2000-2002$ & {$[58,72]$} \\
\hline $\begin{array}{l}\text { PB - Diabetes-Patienten/ } \\
\text { Patienten mit Fettstoffwechsel- } \\
\text { störungen }\end{array}$ & $\begin{array}{l}\text { Klinische Pharmakologie, } \\
\text { Berlin }\end{array}$ & $\begin{array}{l}\text { PB von Patienten mit Fettstoffwechselstörun- } \\
\text { gen und/oder Diabetes mellitus Typ } 2\end{array}$ & 2 & $2000-2003$ & {$[73-74]$} \\
\hline Projekt Neuroderm & $\begin{array}{l}\text { Klinische Pharmakologie, } \\
\text { Berlin }\end{array}$ & PB von Neurodermitis-Patienten & 2 & $2000-2006$ & {$[45,58]$} \\
\hline $\begin{array}{l}\text { PHIT-Studie (PB von Hypertoni- } \\
\text { kern in Thüringen) }\end{array}$ & $\begin{array}{l}\text { LAK Thüringen; Klinische } \\
\text { Pharmakologie, Berlin }\end{array}$ & Verbesserung der AMTS & $1(+/-)$ & 2001 & {$[58,75]$} \\
\hline PB - Asthma-Patienten (2) & ABDA (ZAPP) & Verbesserung der AMTS & 1,4 & $2001-2003$ & {$[76-78]$} \\
\hline PB - Klimakterische Beschwerden & $\begin{array}{l}\text { Klinische Pharmakologie, } \\
\text { Berlin }\end{array}$ & Verbesserung der AMTS & 2 & 2001-2004 & [79] \\
\hline $\begin{array}{l}\text { Case Management für chronisch } \\
\text { Kranke }\end{array}$ & $\begin{array}{l}\text { Klinische Pharmakologie, } \\
\text { Berlin }\end{array}$ & $\begin{array}{l}\text { Implementierung eines Case Managements } \\
\text { (Apotheke) }\end{array}$ & 2 & $2001-2004$ & {$[43,80]$} \\
\hline $\begin{array}{l}\text { PB - Asthma-Patienten im } \\
\text { Kindesalter }\end{array}$ & Universität Würzburg & $\begin{array}{l}\text { Verbesserung von z. B. Lebensqualität und } \\
\text { Compliance }\end{array}$ & 1 & $2002-2006$ & {$[58,81]$} \\
\hline $\begin{array}{l}\text { PB - Alzheimer-Patienten, } \\
\text { Angehörige }\end{array}$ & Freie Universität Berlin & Verbesserung der AMTS & 2 & 2004-2008 & {$[58,82]$} \\
\hline IV-Modell: Gesundes Kinzigtal & $\begin{array}{l}\text { Ärzteinitiative Kinzigtal } \\
\text { e.V., OptiMedis AG }\end{array}$ & $\begin{array}{l}\text { Verbesserung der Patientenversorgung durch } \\
\text { strukturierte Zusammenarbeit }\end{array}$ & 3 & Seit 2005 & [44] \\
\hline $\begin{array}{l}\text { AGnES (Arzt-entlastende, } \\
\text { Gemeinde-nahe, E-Health } \\
\text { gestützte Systemische Intervention) }\end{array}$ & Universität Greifswald & $\begin{array}{l}\text { MA und Reduktion von UAW durch Arzt, } \\
\text { Pflegekraft und Apotheker }\end{array}$ & 2,4 & Seit 2005 & {$[58,83-84]$} \\
\hline ABP-Detektion - Selbstmedikation & ABDA (ZAPP/GB AM) & $\begin{array}{l}\text { Detektion und Lösung von ABP bei } \\
\text { Selbstmedikation }\end{array}$ & 3 & 2007 & {$[58,85]$} \\
\hline ABP-Detektion - Verordnungen & ABDA (ZAPP/GB AM) & $\begin{array}{l}\text { Detektion und Lösung von } \mathrm{ABP} \text { bei } \\
\text { verordneten } \mathrm{AM}\end{array}$ & 3 & 2007 & {$[58,86]$} \\
\hline $\begin{array}{l}\text { Optimierung AM-Versorgung in } \\
\text { Heimen }\end{array}$ & $\begin{array}{l}\text { Klinische Pharmakologie, } \\
\text { Berlin }\end{array}$ & $\begin{array}{l}\text { Identifizierung, Lösung bzw. Vermeidung von } \\
\text { ABP }\end{array}$ & 2 & $2007-2011$ & {$[58,87]$} \\
\hline PB - Geriatrische Patienten & Universität Münster & $\begin{array}{l}\text { ABP-Reduktion, Optimierung der AM-Thera- } \\
\text { pie geriatrischer Heimbewohner }\end{array}$ & 2 & $2008-2011$ & {$[58,88]$} \\
\hline MM - Onkologische Patienten & Universität Bonn & $\begin{array}{l}\text { Interdisziplinäres MM onkologischer Patienten } \\
\text { zur Verbesserung der AMTS }\end{array}$ & 1,4 & $2008-2012$ & {$[58,89-90]$} \\
\hline AMTS 1 in Pflegeheimen & $\begin{array}{l}\text { Universität Witten/ } \\
\text { Herdecke }\end{array}$ & $\begin{array}{l}\text { Bildung von AMTS-Teams (Apotheker, Pflege), } \\
\text { Durchführung von MA }\end{array}$ & $1(-), 6$ & $2009-2010$ & [35] \\
\hline $\begin{array}{l}\text { Häusliches MM - } \\
\text { Diabetes mellitus }\end{array}$ & $\begin{array}{l}\text { Apotheke Krefeld, ABDA } \\
\text { (ZAPP) }\end{array}$ & $\begin{array}{l}\text { Interdisziplinäre Betreuung von Patienten mit } \\
\text { insulinpflichtigem Diabetes mellitus }\end{array}$ & $1(+), 6$ & $2009-2010$ & [37] \\
\hline $\begin{array}{l}\text { Ambulanter } \\
\text { Benzodiazepin-Entzug }\end{array}$ & ABDA & $\begin{array}{l}\text { Interdisziplinäre Betreuung von Patienten } \\
\text { beim Benzodiazepin-Entzug }\end{array}$ & $1(+), 6$ & $2009-2013$ & {$[36,91]$} \\
\hline Fragebogenerhebung & Universität Greifswald & Querschnittsbefragung zur Zusammenarbeit & 7 & 2010 & [30] \\
\hline $\begin{array}{l}\text { PB - Diabetes mellitus- } \\
\text { Patienten }\end{array}$ & Universität Greifswald & $\begin{array}{l}\text { Verbesserung der AMTS eingeschränkt } \\
\text { mobiler Diabetiker in Regionen mit abneh- } \\
\text { mender Versorgungsdichte, Förderung der } \\
\text { interdisziplinären Zusammenarbeit }\end{array}$ & 2,4 & $2010-2013$ & {$[58,92]$} \\
\hline $\begin{array}{l}\text { PB - Morbus Parkinson- } \\
\text { Patienten }\end{array}$ & Universität Münster & $\begin{array}{l}\text { Interdisziplinäre Betreuung von Morbus } \\
\text { Parkinson-Patienten in der Apotheke }\end{array}$ & $2(-), 6$ & $2010-2014$ & [93] \\
\hline care $\mathrm{plus}^{\text {PluV-Modell }}$ & AOK Nordost u. a. & $\begin{array}{l}\text { Etablierung eines geriatrischen Teams zur integ- } \\
\text { rativen Versorgung, Verbesserung der AMTS }\end{array}$ & 1,4 & $2011-2013$ & [94-95] \\
\hline $\begin{array}{l}\text { Qualitätssicherung } \\
\text { Heimversorgung }\end{array}$ & Gesundheitsamt Hamm & $\begin{array}{l}\text { Verbesserung der AMTS und der Zusammen- } \\
\text { arbeit }\end{array}$ & 2,3 & $2012-2013$ & [96] \\
\hline WestGEM & Universität Wuppertal & $\begin{array}{l}\text { Interdisziplinäres MM bei multimorbiden } \\
\text { Patienten }\end{array}$ & $2,4,6$ & $2012-2015$ & {$[31,97]$} \\
\hline $\begin{array}{l}\text { AMTS-AMPEL (AMTS bei Patienten } \\
\text { in Einrichtungen der Langzeitpflege) }\end{array}$ & $\begin{array}{l}\text { Universität Witten/ } \\
\text { Herdecke }\end{array}$ & $\begin{array}{l}\text { AMTS-Teams zur Reduktion von UAW bei } \\
\text { Heimbewohnern (Apotheker, Pflege), struktu- } \\
\text { rierter Austausch mit Ärzten }\end{array}$ & $1,4,6$ & $2012-2015$ & [35] \\
\hline
\end{tabular}


Tab. 5 Fortsetzung.

\begin{tabular}{|c|c|c|c|c|c|}
\hline Titel des Projektes & Wer & Inhalt & Bewertung & Zeitraum & Referenz \\
\hline DelpHi-MV-Studie & $\begin{array}{l}\text { DZNE, Universität } \\
\text { Greifswald }\end{array}$ & Häusliches MM bei Demenzkranken & 1,4 & Seit 2012 & [98-99] \\
\hline Apo-AMTS-Modell & $\begin{array}{l}\text { Universität Münster, } \\
\text { AKWL }\end{array}$ & $\begin{array}{l}\text { Strukturiertes MM zur AMTS-Optimierung bei } \\
\text { Patienten mit Polymedikation }\end{array}$ & 2,4 & Seit 2012 & [100] \\
\hline ATHINA: AMTS in Apotheken & AKNR u. a. & Interdisziplinäre(s) MM und MA & 2,4 & Seit 2012 & [41] \\
\hline $\begin{array}{l}\text { PHARM-CHF (Apothekenbasiertes } \\
\text { interdisziplinäres Programm für } \\
\text { Patienten mit Herzinsuffizienz) }\end{array}$ & ABDA (GB AM), UdS & $\begin{array}{l}\text { Randomisierte, kontrollierte, interdisziplinäre } \\
\text { Studie zur Verbesserung der Therapietreue }\end{array}$ & 1,4 & Seit 2012 & {$[34,101]$} \\
\hline DIADEMA-Studie & Universität Düsseldorf & $\begin{array}{l}\text { Integration eines Apothekers in die } \\
\text { Betreuung jugendlicher Typ-1-Diabetiker }\end{array}$ & $1(+), 6$ & 2013 & {$[40,102]$} \\
\hline Arzneimittelkonto NRW $1 \& 2$ & $\begin{array}{l}\text { CGM, Universität } \\
\text { Bielefeld, Pharmatechnik, } \\
\text { Knappschaft, Lauer- } \\
\text { Fischer }\end{array}$ & $\begin{array}{l}\text { Erstellung eines zentralen Arzneimittelkon- } \\
\text { tos, auf das Heilberufler und Patienten zugrei- } \\
\text { fen können }\end{array}$ & 4 & Seit 2013 & {$[38,103]$} \\
\hline Standardisierte Faxvorlagen & IPP Bremen, AK NDS & $\begin{array}{l}\text { Entwicklung von Faxvorlagen zur Förderung } \\
\text { der Kommunikation }\end{array}$ & 3,7 & Seit 2014 & [29] \\
\hline $\begin{array}{l}\text { PRIMA (Primärsystem-Integration } \\
\text { des MP mit Akzeptanzuntersu- } \\
\text { chung) }\end{array}$ & ABDA (GB AM) & $\begin{array}{l}\text { Gemeinsame Erstellung eines elektronischen } \\
\text { MP, Akzeptanzmessung }\end{array}$ & $1,4,6$ & 2014-2016 & [104] \\
\hline $\begin{array}{l}\text { ARMIN (AM-Initiative Sachsen- } \\
\text { Thüringen) }\end{array}$ & $\begin{array}{l}\text { SAV, ThAV, KVS, KVT, AOK } \\
\text { PLUS, (ABDA/KBV) }\end{array}$ & $\begin{array}{l}\text { Interdisziplinäres MM multimorbider } \\
\text { Patienten, Förderung der Zusammenarbeit }\end{array}$ & $1,4,6$ & Seit 2014 & [16] \\
\hline AMTS in der Kommunikation & $\begin{array}{l}\text { Apothekerstiftung } \\
\text { Westfalen-Lippe }\end{array}$ & $\begin{array}{l}\text { Befragung, u. a. zu AMTS im Alltag, } \\
\text { Rollenverständnis }\end{array}$ & 3,7 & 2015 & [105-106] \\
\hline $\begin{array}{l}\text { MMP16 (Metropol-Medikations- } \\
\text { plan) }\end{array}$ & $\begin{array}{l}\text { Klinikum Fürth, Zentrale } \\
\text { Notaufnahme }\end{array}$ & $\begin{array}{l}\text { Interdisziplinäre Erstellung und Aktualisie- } \\
\text { rung des MP }\end{array}$ & 1,4 & $2015-2016$ & [107] \\
\hline Tandemvisite in Heimen & $\begin{array}{l}\text { Institut für Allgemeinmedi- } \\
\text { zin, MHH, AK NDS }\end{array}$ & $\begin{array}{l}\text { Interdisziplinäre MM durch Apotheker, Pflege } \\
\text { und Ärzte }\end{array}$ & $1(-)$ & $2015-2016$ & [108] \\
\hline Talk Smart & $\begin{array}{l}\text { Universitätsklinikum } \\
\text { Heidelberg }\end{array}$ & $\begin{array}{l}\text { Befragung: Erhebung wichtiger Faktoren der } \\
\text { Zusammenarbeit }\end{array}$ & $1(+), 7$ & $2015-2016$ & [5] \\
\hline $\begin{array}{l}\text { InTherAKT (Initiative zur AMTS in } \\
\text { der Altenhilfe durch Kooperation } \\
\text { und Teamwork) }\end{array}$ & $\begin{array}{l}\text { Paracelsus Medizinische } \\
\text { Privatuniversität, } \\
\text { Salzburg, Partner in } \\
\text { Deutschland }\end{array}$ & $\begin{array}{l}\text { Therapieoptimierung bei Heimbewohnern, } \\
\text { interdisziplinäre Fallkonferenzen }\end{array}$ & 4,7 & Seit 2015 & [33] \\
\hline AMBER-Studie & $\begin{array}{l}\text { Öffentliche Apotheke, } \\
\text { Münster }\end{array}$ & $\begin{array}{l}\text { Interdisziplinäre Leitfadenentwicklung für das } \\
\text { MM in Altenheimen }\end{array}$ & 4,7 & $2016-2018$ & [109] \\
\hline Medikationskonsil Greifswald & $\begin{array}{l}\text { KVMV, AVMV, AOK } \\
\text { Nordost, Ärztenetz } \\
\text { Greifswald }\end{array}$ & $\begin{array}{l}\text { Ärzte verordnen Medikationskonsil durch eine } \\
\text { Apotheke }\end{array}$ & 4 & Seit 2016 & [110] \\
\hline $\begin{array}{l}\text { SiMbA-Projekt (Studienprotokoll } \\
\text { publiziert) }\end{array}$ & $\begin{array}{l}\text { Paracelsus Medizinische } \\
\text { Privatuniversität, Salzburg }\end{array}$ & $\begin{array}{l}\text { AMTS-Onlineschulungen, Onlineplattform } \\
\text { zum Informationsaustausch, interprofessio- } \\
\text { nelles MM }\end{array}$ & 1, 5, 6 (geplant) & Start 2017 & [111] \\
\hline
\end{tabular}

Bewertung: ${ }^{1}$ Die Zusammenarbeit ist vorgeschrieben; ${ }^{2}$ Die Zusammenarbeit ist optional: Der Heilberufler (z. B. der Apotheker) entscheidet im Einzelfall, ob er das Problem alleine bzw. mit dem Patienten lösen kann, den anderen Heilberufler (z. B. Arzt) kontaktiert oder den Patienten zurück (z. B. in die Arztpraxis) schickt (z. B. Projekte zur PB); Bewertung: (+)= insgesamt positive bzw. harmonische Zusammenarbeit, (-)=Zusammenarbeit wurde negativ bewertet bzw. verlief schwierig; ${ }^{3}$ Keine direkte Zusammenarbeit; ${ }^{4}$ Es liegen (noch) keine umfassenden Informationen vor, ob bzw. wie die Heilberufler zusammenarbeiteten. Insbesondere bei noch nicht bzw. erst kürzlich abgeschlossenen Projekten, werden diese Ergebnisse noch erwartet; ${ }^{5}$ Die Arzt-ApothekerZusammenarbeit bzw. der Informationsaustausch wurde (teilweise) prospektiv geplant, standardisiert durchgeführt oder strukturiert ausgewertet; ${ }^{6}$ Befragungen der Heilberufler (z. B. anhand eines Fragebogens, interdisziplinäre Gesprächsrunden), um deren Perspektiven zur Zusammenarbeit zu erfassen; Abkürzungen: (Landes-) Apothekerkammer (L-)AK, AK Niedersachsen AK NDS, AK Nordrhein AKNR, AK Westfalen-Lippe AKWL, Apothekerverband $A V$, Arzneimittelbezogene Probleme ABP, Arzneimitteltherapiesicherheit AMTS, Bundesvereinigung Deutscher Apothekerverbände ABDA, CompuGroup Medical CGM, Deutsches Zentrum für Neurodegenerative Erkrankungen DZNE, Geschäftsbereich Arzneimittel (der ABDA) GB AM, Institut für Public Health und Pflegeforschung IPP, Kassenärztliche Vereinigung KV, Medikationsanalyse MA, Medikationsmanagement MM, Medikationsplan MP, Thüringer AV ThAV, KV Thüringen $K V T$, Medizinische Hochschule Hannover $M H H$, Mecklenburg-Vorpommern MV, Optimierte Arzneimittelversorgung OAV, Pharmazeutische Betreuung PB, Sächsischer AV SAV, Sächsische LAK SLAK, Technische Universität TU, Unerwünschte Arzneimittelwirkungen UAW, Universität des Saarlandes UdS, Westphalian study on a medication therapy management and home care based intervention under gender specific aspects in elderly multimorbid patients WESTGEM, Zentrum für Arzneimittelinformation und Pharmazeutische Praxis (der ABDA) ZAPP 
mär adäquat vergütet wird [39]. Neben der materiellen Incentivierung ist auch eine ideelle Incentivierung möglich, d. h. dass die Heilberufler durch den Austausch von Information profitieren oder ihre Zufriedenheit durch eine Verbesserung der Patientenversorgung erhöht wird [40].

Sowohl die Machbarkeit als auch eine adäquate Incentivierung sind Voraussetzung für eine erfolgreiche Dissemination [33,4143] und vereinfachen die nachhaltige Integration der Projekte in bestehende Versorgungsstrukturen in der Routineversorgung [42, 44, 45]. Dies wird auch durch die Ergebnisse internationaler Projekte gespiegelt, bei denen zusätzliche Dienstleistungen typischerweise einen Weg in die Routinevergütung gefunden haben $[7,8]$.

Zuletzt sind geeignete Untersuchungs- und Bewertungskriterien, bspw. in Form valider Messinstrumente, wichtig, um diese Erfolge auch „messbar“ und somit für beide Seiten sichtbar zu machen. Bis heute fehlen standardisierte Messmethoden, um das Ausmaß, den Gewinn und den Aufwand der Arzt-ApothekerZusammenarbeit zu messen. In einigen Projekten wurden spezifische Fragebögen, z. B. zur Zufriedenheit [36, 42] entwickelt, die jedoch nur schlecht auf andere Settings übertragen werden können. Dabei würden solche standardisierten Messmethoden nicht nur die Evaluation von (Forschungs-)Projekten ermöglichen, sondern sie könnten auch dem kontinuierlichen Benchmarking in der Routineversorgung dienen. Anhand von Fragebögen könnten bspw. Variablen wie Teilnehmerzufriedenheit, Zeitersparnis, Wissenszunahme und der Wunsch bzw. die Bereitschaft das Projekt fortzuführen erhoben werden. Diese Ergebnisse würden zudem eine Vergleichbarkeit der Projekte ermöglichen und könnten bei der Durchführung zukünftiger Projekte berücksichtigt werden.

\section{Limitationen}

Die systematische Suche in den Literaturdatenbanken PubMed und Psyclnfo ergab nur wenige Treffer, wohingegen in den übrigen Informationsquellen zahlreiche thematisch passende Artikel identifiziert werden konnten. Diese Quellen unterstützen keine umfassende strukturierte Suche, weswegen diese Übersichtsarbeit trotz aufwändiger Literatursuche keine Vollständigkeit garantieren kann. Jedoch ermöglichte diese erweiterte Suche die Identifikation zahlreicher Projekte und Initiativen, die nur lokal oder regional durchgeführt wurden und auch ohne konsequente wissenschaftliche Begleitevaluation Hinweise zum Stand der Arzt-Apotheker-Zusammenarbeit in Deutschland aufzeigen. Die über den Innovationsfonds geförderten Projekte zur interprofessionellen Zusammenarbeit laufen gerade erst an; erste Ergebnisse werden in den kommenden Jahren erwartet.

Eine weitere Limitation stellt die variable Qualität der Berichterstattung der Projekte dar. Oftmals ist die interprofessionelle Zusammenarbeit eher ein Nebeneffekt, der selten speziell evaluiert und noch seltener umfassend berichtet wird. So gibt es sicherlich weitaus mehr Projekte zur Arzneimitteltherapiesicherheit, die jedoch hier nicht berichtet werden, da die Arzt-Apotheker-Zusammenarbeit in den Publikationen nicht explizit diskutiert wird.

Insgesamt sind die Projekte nur sehr eingeschränkt vergleichbar, da diese Übersichtsarbeit sowohl wissenschaftliche Studien als auch kleinere Initiativen umfasst, die gewissermaßen nebenbei im
Alltag entstanden sind. Gleichzeitig bildet die Arbeit damit aber auch den Status Quo bisheriger Projektverläufe ab und unterstreicht den potentiellen Nutzen von Standards für die Berichterstattung.

\section{Schlussfolgerung}

In dieser Arbeit wurden insgesamt 6 Faktoren herausgearbeitet, die eine erfolgreiche Zusammenarbeit von Arzt und Apotheker im ambulanten Versorgungssektor begünstigen. Grundvoraussetzung erscheint dabei das gegenseitige Kennenlernen, die Kenntnis der Kompetenzen des anderen und ein offenes Miteinander (I). Erfolgreiche Projekte zeichneten sich darüber hinaus aus durch das Einbeziehen beider Heilberufler in die Projektplanung (II), die Etablierung gemeinsamer Informationsveranstaltungen (III), die Sicherstellung der (technischen) Machbarkeit (IV) durch ideelle bzw. materielle Incentivierung $(\mathrm{V})$ sowie die Einbindung in bestehende oder der Aufbau neuer Versorgungsstrukturen (VI).

\section{Finanzielle Unterstützung}

Die Erstellung dieser Arbeit wurde finanziell unterstützt durch die ABDA - Bundesvereinigung Deutscher Apothekerverbände e.V.

\section{Interessenskonflikt}

Die Autoren geben an, dass kein Interessenkonflikt besteht.

\section{Literatur}

[1] Hirsch JD, Steers N, Adler DS et al. Primary care-based, pharmacistphysician collaborative medication-therapy management of hypertension: a randomized, pragmatic trial. Clin Ther 2014; 36: 1244-1254

[2] Sisson EM, Dixon DL, Kildow DC et al. Effectiveness of a pharmacistphysician team-based collaboration to improve long-term blood pressure control at an inner-city safety-net clinic. Pharmacotherapy 2016; 36: 342-347

[3] Teinilä T, Kaunisvesi K, Airaksinen M. Primary care physicians' perceptions of medication errors and error prevention in cooperation with community pharmacists. Res Social Adm Pharm 2011; 7 : 162-179

[4] Kelly DV, Bishop L, Young S et al. Pharmacist and physician views on collaborative practice: Findings from the community pharmaceutical care project. Can Pharm J (Ott) 2013; 146: 218-226

[5] Weissenborn M, Haefeli WE, Peters-Klimm F et al. Interprofessional communication between community pharmacists and general practitioners: a qualitative study. Int J Clin Pharm 2017; 39: 495-506

[6] van Mil JW. Pharmaceutical care in community pharmacy: practice and research in the Netherlands. Ann Pharmacother 2005; 39: $1720-1725$

[7] Costa D, Van C, Abbott P et al. Investigating general practitioner engagement with pharmacists in Home Medicines Review. J Interprof Care 2015; 29: 469-475

[8] Rodgers ], Walker R. Medicines use reviews: what they are for and how to make use of them. Br J Community Nurs 2007; 12: 563-565 
[9] Tonna AP, Stewart D, West B et al. Pharmacist prescribing in the UK - a literature review of current practice and research. J Clin Pharm Ther 2007; 32: 545-556

[10] Tsuyuki RT, Houle SK, Charrois TL et al. Randomized Trial of the Effect of Pharmacist Prescribing on Improving Blood Pressure in the Community: The Alberta Clinical Trial in Optimizing Hypertension (RxACTION). Circulation 2015; 132: 93-100

[11] Prisma Guideline. Im Internet: http://www.prisma-statement.org/; Stand: 16.01.2018

[12] PZ-Nachrichten. Kiefer: AMTS braucht zwei Säulen. Im Internet: http://www.pharmazeutische-zeitung.de/index.php?id = 61640; Stand: 16.01 .2018

[13] Richter-Kuhlmann E. Arzneimittelversorgung: Der Arzt als Schlüsselfigur. Dtsch Arztebl 2014; 111: (48): A-2101

[14] Bardet JD, Vo TH, Bedouch P et al. Physicians and community pharmacists collaboration in primary care: A review of specific models. Res Social Adm Pharm 2015; 11: 602-622

[15] Döhler N, Krolop L, Ringsdorf S et al. Task allocation in cancer medication management - integrating the pharmacist. Patient Educ Couns 2011; 83: 367-374

[16] ARMIN Projekt. Im Internet: http://www.arzneimittelinitiative.de/ ueber-armin/aktuelle-nachrichten/; Stand: 16.01.2018

[17] Apothekerkammer Sachsen-Anhalt. Magdeburger Medizinstudenten in der Apotheke. Dtsch Apoth Ztg 2016; 156: 108

Bundesverband der Pharmaziestudierenden in Deutschland e.V. Meinungspapier: Intensivierung der Zusammenarbeit von Studierenden der Gesundheitsberufe. Im Internet: http://www.bphd.de/ mitmischen-statt-nur-moersern/meinungspapiere/; Stand: 16.01.2018

[18] Homepage Aktionsbündnis Patientensicherheit. Im Internet: http:// www.aps-ev.de/; Stand: 16.01.2018

[19] Ärztezeitung: Auffälligkeiten zu thematisieren, darf kein Tabu sein. Im Internet: http://www.aerztezeitung.de/praxis_wirtschaft/rezepte/ article/873196/arzneiverordnung-auffaelligkeiten-thematisierendarf-kein-tabu.html; Stand: 16.01.2018

[20] Ärztezeitung: Intensivere Kooperation mit Apothekern. Im Internet: http://www.aerztezeitung.de/praxis_wirtschaft/netze_kooperation/ article/898367/westfalen-lippe-intensivere-kooperation-apothekern. html; Stand: 16.01.2018

[21] Sachsen: Ärzte und Apotheker wollen enger kooperieren. Im Internet: https://www.aerzteblatt.de/nachrichten/72804/Sachsen-Aerzte-undApotheker-wollen-enger-kooperieren; Stand: 16.01.2018

[22] Gerlof H. Vernetzung im Dienste der Patienten. Im Internet: http:// www.aerztezeitung.de/praxis_wirtschaft/praxismanagement/ praxisfuehrung/article/858564/diabetologe-dr-michael-boehmervernetzung-dienste-patienten.html?sh = 55\&h=-2095568945; Stand: 16.01.2018

[23] Ditzel P. Eine Apothekerin, die bewegt. Dtsch Apoth Ztg 2016; $156: 72$

[24] DAZ-Redaktion. Arzt fragt Apotheker. Dtsch Apoth Ztg 2013; 153: 72

[25] Gemeinsamer Bundesausschuss. Innovationsfonds. Geförderte Projekte „Neue Versorgungsformen“. Im Internet: https:// innovationsfonds.g-ba.de/projekte/neue-versorgungsformen; Stand: 10.04.2017

[26] Aktionsplan des Bundesministeriums für Gesundheit. Im Internet: www.ap-amts.de; Stand: 16.01.2018

[27] Faller C, Haefeli WE, Seidling HM. How to move an elephant. Dtsch Apoth Ztg 2016; 156: 56

[28] Willers ], Hoffmann F, Götz S et al. Kommunikation zwischen Arzt und Apotheker. Im Internet: http://www.pharmazeutische-zeitung.de/ index.php?id = 53765; Stand: 16.01.2018

[29] Wüstmann AF, Haase-Strey C, Kubiak T et al. Cooperation between community pharmacists and general practitioners in eastern Germany: attitudes and needs. Int J Clin Pharm 2013; 35: 584-592
[30] Rose O, Mennemann $\mathrm{H}$, John C et al. Priority setting and influential factors on acceptance of pharmaceutical Recommendations in Collaborative Medication Reviews in an Ambulatory Care Setting Analysis of a Cluster Randomized Controlled Trial (WestGem-Study). PLoS One 2016; 11: e0156304

[31] Ausbildung. Grünes Licht für Leipzig. Dtsch Apoth Ztg 2016; 156: 28

[32] Mahlknecht A, Nestler N, Bauer U et al. Effect of training and structured medication review on medication appropriateness in nursing home residents and on cooperation between health care professionals: the InTherAKT study protocol. BMC Geriatr 2017; 17: 24

[33] Fikenzer K, Rettig-Ewen V, Saar M et al. Einnahmetreue - Herausforderung und Chance bei der medikamentösen Therapie der chronischen Herzinsuffzienz. Pharm-CHF: Multizentrische Studie zur Verbesserung der Einnahmetreue bei Patienten mit chronischer Herzinsuffizienz startet demnächst im Saarland. Saarländisches Ärzteblatt 2012; 8: 17-18

Abschlussbericht zum Projekt ArzneiMitteltherapiesicherheit bei Patienten in Einrichtungen der Langzeitpflege (AMTS-AMPEL). Im Internet: https://www.amts-ampel.de/publikationen/; Stand: 16.01.2018

ABDA. Sachbericht: Ambulanter Entzug Benzodiazepin-abhängiger Patientinnen und Patienten in Zusammenarbeit von Apotheker und Hausarzt. Im Internet: http://www.abda.de/benzoprojekt.html; Stand: 16.01 .2018

[34] Krüger M, Griese N, Schulz M. Medikationsmanagement für Menschen mit Diabetes. Diabetes Stoffw Herz 2011; 20: 219-226

[35] Homepage Arzneimittelkonto NRW. Im Internet: https://www. arzneimittelkonto-nrw.de/de/index.de.jsp; Stand: 16.01.2018

[36] Van Mil JWF, de Boer WO, Tromp TFJ. European barriers to the implementation of pharmaceutical care. Int J PharmPract 2001; 9: 163-167

[37] Obarcanin E, Krüger M, Müller $P$ et al. Pharmaceutical care of adolescents with diabetes mellitus type 1: the DIADEMA study, a randomized controlled trial. Int J Clin Pharm 2015; 37: 790-798

[38] Seidling HM, Send AF, Bittmann J et al. Medication review in German community pharmacies - Post-hoc analysis of documented drug-related problems and subsequent interventions in the ATHINA-project. Res Social Adm Pharm 2016; 13: 1127-1134

[39] Jaehde U, Thürmann PA. Medication safety in nursing homes. Z Evid Fortbild Qual Gesundhwes 2012; 106: 712-716

Schäfer M, Schmid-Sroka D.Kurzfassung des Endberichtes. Implementierung und Evaluation eines Case Managements für chronisch kranke Patienten in öffentlichen Apotheken. 2004. Im Internet: http://www. beta-institut.de/files/betalnstitut/downloads/CM_Apotheke_ Kurzbericht.pdf; Stand: 16.01.2018

[40] Gesundes Kinzigtal. GeKiM. DRKS-ID DRKS00004283. Im Internet: http://www.drks.de/drks_web/; Stand: 16.01.2018

[41] Büke K. Analyse und Bewertung der Pharmazeutischen Betreuung von Patienten mit chronischen Hautkrankheiten Schwerpunkt Neurodermitis in der öffentlichen Apotheke [Dissertation]. Humboldt-Universität Berlin, 2006

[42] Aus Kammern und Verbänden. Neues zur Qualitätssicherung dermatologischer Rezepturen. Dtsch Apoth Ztg 2010; 150: 78

[43] Brandenburgischer Apotheker- und Ärztetag. Im Internet: http:// www.aerztezeitung.de/praxis_wirtschaft/rezepte/article/860866/ apotheker-arzt-kooperation-a-o.html?sh = 26\&h = -1222579484; Stand: 10.04.2017

[44] Müller-Mundt G, Schaeffer D, Haslbeck J. Alltagsnahe Förderung des Selbstmanagements komplexer Medikamentenregime bei chronischer Krankheit - Bedeutung und Ansatzpunkte eines pflegerischen Interventionskonzepts. Meeting Abstract. German Medical Science GMS Publishing House; 2007. Doc07gmds639 
[45] [Anonym]. Arzt-Apotheker-Dialog. Dtsch Apoth Ztg 2016; 156: 79

[46] Apothekerkammer Nordrhein. Informationskonzept Medikationsplan. Im Internet: https://www.aknr.de/presse/presseinfo. php?id = 43\&pid = 90\&sid = 3r9uhplukbsbkq1 kq08eb8iv21; Stand: 10.04.2017

[47] Sapremo. Gefördertes Projekt der Robert Bosch Stiftung. Im Internet: http://www.bosch-stiftung.de/content/language1/html/65672.asp; Stand: 14.11.2017

[48] Gerlach N, Michiels-Corsten M, Schleef T et al. Exploration zu Absetzstrategien bei Patienten mit Polypharmazie: Eine interdisziplinäre Perspektive. Meeting Abstract. GMS Publishing House; 2017. Doc17degam025

[49] Pulst A, Hoffmann F, Dehlfing A et al. Verbesserung der Arzneimitteltherapiesicherheit bei Pflegeheimbewohnern mit chronischer Niereninsuffizienz. Meeting Abstract. GMS Publishing House; 2017. Doc17degam130

[50] Homepage Teddybärenklinik. Im Internet: https://teddyklinikhd. wordpress.com/team/; Stand: 10.04.2017

[51] BPhD e.V. Pharmaweekend. Im Internet: http://www.bphd.de/ ueber-uns/pharmaweekend/; Stand: 16.01.2018

[52] Bundeskongress der Medizinstudierenden. Im Internet: http://www. pharmazeutische-zeitung.de/index.php?id=32172; Stand: 16.01.2018

[53] Gensthaler BM. Vom Fachkollegen lernen. Im Internet: http://www. pharmazeutische-zeitung.de/index.php?id=21680; Stand: 16.01.2018

[54] Projektdatenbank: Förderinitiative Pharmazeutische Betreuung. Im Internet: http://www.foerderinitiative.de/projektdatenbank.html; Stand: 16.01.2018

[55] Ney R. Im Team für gesunde Ernährung. Im Internet: http://www. aerztezeitung.de/ extras/extras_specials/apothekerplus/ article/675002/team-gesunde-ernaehrung.html; Stand: 16.01.2018

[56] Grossmann U.Mit Fachwissen und Fingerspitzengefühl. Im Internet: http://www.pharmazeutische-zeitung.de/index.php?id = 40207; Stand: 16.01 .2018

[57] Grummel R, Greitemeier A. The 7+ project: Improving patient safety by facilitating communication among health professionals in Gelsenkirchen. Meeting Abstract. German Medical Science GMS Publishing House; 2016. Doc16gaa21

[58] Homepage IDH. Im Internet: http://www.idh-sh.de/; Stand: 16.01.2018

[59] ABDA. Apotheker und Ärzte: Leitfaden zur Zusammenarbeit. Pharm Ztg 1998; 143: 48 51-52

[60] Homepage EADV. Im Internet: http://www.deutsche-diabetesgesellschaft.de/ueber-uns/ausschuesse-und-kommissionen/ kommission-zur-einbindung-der-apotheker-in-diediabetikerversorgung.html; Stand: 16.01.2018

[61] Homepage Kinderstube. Im Internet: http://www.kinderstubesachsen.de; Stand: 16.01 .2018

[62] DAZ-Aktuell. Kooperation für bessere Compliance. Dtsch Apoth Ztg 2011; 151: 41

[63] Vernetzte Arzneimitteltherapiesicherheit. Im Internet: https:// msagd.rlp.de/de/service/presse/detail/news/detail/News/ vernetzte-arzneimitteltherapiesicherheit-mit-unterstuetzungeines-elektronischen-medikationsplans-in/; Stand: 16.01.2018

[64] Ärzte und Apotheker in Baden-Württemberg kooperieren im Notdienst. Im Internet: https://www.aerzteblatt.de/ treffer?mode $=s \& w o=17 \&$ typ $=1 \&$ nid $=65204 \& s=$ apotheke; Stand: 16.01.2018

[65] Schulz M, Verheyen F, Mühlig S et al. Pharmaceutical care services for asthma patients: a controlled intervention study. J Clin Pharmacol 2001; 41: 668-676
[66] Hämmerlein A, Müller U, Schulz M. Versorgungsmanagement für Menschen mit Asthma - Einbindung der Apotheker. Z Evid Fortbild Qual Gesundh Wesen 2010; 104: 92-98

[67] Bernsten C, Björkman I, Caramona M et al. Improving the well-being of elderly patients via community pharmacy-based provision of pharmaceutical care: a multicentre study in seven European countries. Drugs Aging 2001; 18: 63-77

[68] Uferer Ch, Grappweis J, Schubert I. Pharmazeutische Betreuung von Schmerzpatienten. Pharm Ztg 2002; 147: 27-31

Birnbaum U. Analyse und Bewertung der Pharmazeutischen Betreuung von Patienten mit Fettstoffwechselstörungen und/oder Diabetes mellitus Typ 2 in der öffentlichen Apotheke: Möglichkeiten und Grenzen [Dissertation]. Humboldt-Universität Berlin, 2004

[69] Birnbaum U, Schaefer M, Verheyen F et al. LipoPharm-Projekt. Pharmazeutische Betreuung von Patienten mit Fettstoffwechselstörungen. Pharm Ztg 2003; 148: 3848-3853

Goebel R. Analyse und Bewertung der Pharmazeutischen Betreuung medikamentös therapierter Hypertoniker in der öffentlichen Apotheke [Dissertation]. Humboldt-Universität Berlin, 2002

[70] Hämmerlein A, Müller U, Schulz M. Pharmacist-led intervention study to improve inhalation technique in asthma and COPD patients. J Eval Clin Pract 2011; 17: 61-70

[71] Hämmerlein A, Müller U, Schulz M. Versorgungsmanagement für Menschen mit Asthma - Einbindung der Apotheker. Z Evid Fortbild Qual Gesundh Wesen 2010; 104: 92-98

[72] Mangiapane S, Mühlig S, Ihle P et al. Pharmazeutische Betreuung: Resultate der Asthma-Studie im KV-Bezirk Trier. Pharm Ztg 2005; 150: $108-115$

Hommel F. Zur Betreuung von Frauen mit klimakterischen Beschwerden in der öffentlichen Apotheke - Möglichkeiten und Grenzen [Dissertation]. Humboldt-Universität Berlin, 2005

Schmid-Sroka D. Implementierung und Evaluation eines Case Management für chronisch kranke Patienten in öffentlichen Apotheken [Dissertation]. Charité Universitätsmedizin Berlin, 2005

Thern J. Pharmazeutische Betreuung von Kindern mit Asthma bronchiale in ambulanter Therapie [Dissertation]. Bayerische Julius-Maximilians-Universität Würzburg, 2006

[73] Schwalbe O, Scheerans C, Freiberg I et al. Compliance assessment of ambulatory Alzheimer patients to aid therapeutic decisions by healthcare professionals. BMC Health Serv Res 2010; 10: 232

[74] Fiß T, Meinke-Franze C, van den Berg N et al. Effects of a three party healthcare network on the incidence levels of drug related problems. Int J Clin Pharm 2013; 35: 763-771

[75] van den Berg N, Fiss T, Meinke C et al. GP-support by means of AGnES-practice assistants and the use of telecare devices in a sparsely populated region in Northern Germany - proof of concept. BMC. Fam Pract 2009; 10: 44

[76] Eickhoff C, Hämmerlein A, Griese $\mathrm{N}$ et al. Nature and frequency of drug-related problems in self-medication (over-the-counter drugs) in daily community pharmacy practice in Germany. Pharmacoepidemiol Drug Saf 2012; 21: 254-260

[77] Nicolas A, Eickhoff C, Griese $N$ et al. Drug-related problems in prescribed medicines in Germany at the time of dispensing. Int I Clin Pharm 2013; 35: 476-482

Urhahn T. Optimierung der Arzneimittelversorgung für Bewohner von Alten- und Pflegeheimen durch Identifizierung und Lösung bzw. die prophylaktische Vermeidung arzneimittelbezogener Probleme [Dissertation]. Humboldt-Universität Berlin, 2006

Kruse J. Optimierung der Versorgung geriatrischer Bewohner von Alten- und Pflegeheimen durch intensive Pharmazeutische Betreuung [Dissertation]. Westfälische Wilhelms-Universität Münster, 2012 
[78] Krolop L, Ko YD, Schwindt PF et al. Adherence management for patients with cancer taking capecitabine: a prospective two-arm cohort study. BMJ Open 2013; 3: pii: e003139

[79] Krolop L. Adherence Management for Cancer Patients on Capecitabine [Dissertation]. Rheinische Friedrich-Wilhelms-Universität Bonn 2013

[80] Eckert-Lill C, Holzbach R, Möbius JR et al. Betreuung durch Apotheker und Arzt. Im Internet: http://www.pharmazeutische-zeitung. de/?id = 52289; Stand: 16.01 .2018

Hebert AF. Pilotstudie zur Pharmazeutischen Betreuung eingeschränkt mobiler Patienten in Regionen mit abnehmender Versorgungsdichte [Dissertation]. Ernst-Moritz-Arndt-Universität Greifswald, 2015

[81] Henrichsmann M, Hempel G. Impact of medication therapy management in patients with Parkinson's disease. Int J Clin Pharm 2016; 38: 54-60

[82] Hanke F, Hildebrand J, Joks G et al. Prävention arzneimittelassoziierter Erkrankungen bei stationären Altenheimbewohnern durch ein pflegezentriertes Risikomanagement im Rahmen einer Integrativen Versorgung. Meeting Abstract. German Medical Science GMS Publishing House; 2013. Doc13gaa30

[83] Hanke F, Joks G, Hildebrand J et al. Aufbau und Evaluation von Arzneimittelrisikomanagementsystemen in Altenheimen. Meeting Abstract. German Medical Science GMS Publishing House; 2013. Doc13gaa05

[84] Stapel U.Teilen von Tabletten: Qualitätssicherung in der Heimversorgung. Im Internet: http://www.pharmazeutische-zeitung.de/index. php?id= 52396; Stand: 10.04.2017

[85] Köberlein-Neu J, Mennemann H, Hamacher S et al. Interprofessionelles Mediaktionsmanagement bei multumorbiden Patienten - Eine Cluster-randomisierte Studie (WestGem-Studie). Dtsch Ärztebl Int 2016; 113: 741-748

[86] Thyrian JR, Winter P, Eichler T et al. Relatives' burden of caring for people screened positive for dementia in primary care: Results of the DelpHi study. Z Gerontol Geriatr 2017; 50: 4-13

[87] Eichler T, Thyrian JR, Hertel ] et al. Rates of formal diagnosis in people screened positive for dementia in primary care: results of the DelpHi-Trial. J Alzheimers Dis 2014; 42: 451-458

[88] Apo-AMTS-Modell. Im Internet: http://www.mgepa.nrw.de/ mediapool/pdf/gesundheit/gesundheitspreis_2013/ Projektbeschreibung_2_Preis.pdf; Stand: 16.01.2018
[89] Laufs U, Böhm M, Kroemer HK et al. Strategien zur Verbesserung der Einnahmetreue von Medikamenten. Dtsch Med Wochenschr 2011; 1369: 1616-1621

Obarcanin E. Pharmaceutical Care of Adolescents with Type 1 Diabetes Mellitus: DIADEMA Trial: A Randomized Controlled Trial [Dissertation]. Heinrich-Heine-Universität Düsseldorf, 2014

[90] Schlingensiepen I. Arzneimittelkonto soll Pfleger und Apotheker einbinden. Im Internet: http://www.aerztezeitung.de/praxis_ wirtschaft/rezepte/article/915017/nrwarzneimittelkonto-sollpfleger-apotheker-einbinden.html?sh = 1\&h=1036803025; Stand: 16.01.2018

[91] PRIMA. Im Internet: https://www.abda.de/uploads/tx_news/ PRIMA_Kurzfassung_2014-01-28.pdf; Stand: 16.01.2018

[92] Lege artis. Kommunikationsherausforderungen an der Schnittstelle Arzt - Apotheker. Im Internet: http://www.akwl.de/ apothekerstiftung/inhalt.php?lid=897; Stand: 16.01.2018

Bennhoff K. AMTS in the communication between physicians and pharmacists. Meeting Abstract. German Medical Science GMS Publishing House; 2016; Doc16gaa12

[93] Homepage MMP16. Im Internet: http://www.emedikationsplan.de/; Stand: 16.01 .2018

Niedersächsisches Forschungsprojekt zu Multimedikation in Pflegeheimen: Posterpreis für interdisziplinäres Medikationsmanagement. Im Internet: http://www.apothekerkammer-niedersachsen.de/ presse.php?view $=\% 7$ C3042,4; Stand: 16.01 .2018

[94] Rose O, Erzkamp S. Development and evaluation of an algorithm in medication management for best practice. Effectiveness of the intervention and translation into standard care for nursing home residents - AMBER-Study. Meeting Abstract. German Medical Science GMS Publishing House; 2016. Doc16gaa16

[95] Schnack D.Medikationskonsil geht an den Start. Im Internet: http:// www.aerztezeitung.de/politik_gesellschaft/krankenkassen/ article/906346/praxisnetz-greifswald-medikationskonsil-geht-start. html Stand: 16.01.2018

[96] Schaffler-Schaden D, Schreier M, Pitzer S et al. Optimierung der Sicherheit in der Medikamententherapie bei Altenheimbewohnern Das SiMbA-Projekt: Studienprotokoll und Baselinedaten. Meeting Abstract. GMS Publishing House; 2017. Doc17degam132 